\title{
TIC et parrainage dans les mouvements militants de défense des sans-papiers en France
}

Dana Diminescu et Matthieu Renault

\section{Q OpenEdition \\ 1 Journals}

Édition électronique

URL : http://journals.openedition.org/ticetsociete/613

DOI : 10.4000/ticetsociete.613

Éditeur

Association ARTIC

Référence électronique

Dana Diminescu et Matthieu Renault, «TIC et parrainage dans les mouvements militants de défense des sans-papiers en France », tic\&société [En ligne], Vol. 3, n 1-2| 2009, mis en ligne le 12 décembre 2009, consulté le 20 avril 2019. URL : http://journals.openedition.org/ticetsociete/613 ; DOI : 10.4000/ ticetsociete.613 


\title{
TIC et parrainage dans les mouvements militants de défense des sans-papiers en France
}

\author{
Dana Diminescu \\ École d'ingénieur Télécom ParisTech \\ 46, rue Barrault, 75013 Paris \\ Programme TIC-Migrations (Fondation Maison des Sciences de l'Homme) \\ 54, boulevard Raspail, 75006 Paris \\ diminesc@msh-paris.fr

\section{Matthieu Renault} \\ Programme TIC-Migrations (Fondation Maison des Sciences de l'Homme) \\ 54, boulevard Raspail, 75006 Paris \\ matthieu.renault@gmail.com
}

Dana Diminescu est sociologue, enseignant-chercheur au département sciences économiques et sociales de Telecom ParisTech Paris et directrice scientifique du programme TIC-Migrations, créé en 2003 à la Fondation Maison des sciences de l'Homme de Paris-Raspail. Ses recherches portent sur les pratiques de communication et les usages des technologies de l'information et de la communication en situation de mobilité, en particulier leurs usages par les migrants, et sur l'occupation des territoires numériques par les diasporas (projet «E-Diasporas Atlas »). Parmi ses publications, voir " Le migrant connecté Pour un manifeste épistémologique ", <http://ssi.sagepub.com/cgi/ content/french abstract/47/4/565/>.

Matthieu Renault est ingénieur de recherche au département sciences économiques et sociales de Telecom ParisTech et attaché de recherche dans le programme TIC et Migrations de la Fondation Maison des sciences de l'Homme de Paris-Raspail. II est doctorant en philosophie à l'École des hautes études en sciences sociales (EHESS, Paris) sur «Frantz Fanon et les langages postcoloniaux ». Ses recherches dans le domaine des "TIC et migrations" portent sur l'occupation des territoires numériques par les diasporas (projet «EDiasporas Atlas »), le web matrimonial des migrants, l'usage des plateformes sociales (web 2.0) par les migrants et les pratiques des TIC dans les mouvements de sans-papiers. 


\title{
TIC et parrainage dans les mouvements militants de défense des sans-papiers en France
}

\begin{abstract}
Résumé
Cet article a pour objet les usages d'internet - et tout particulièrement des listes de discussion - dans le Réseau Éducation sans frontières, réseau de soutien aux sans-papiers. Ces usages sont étroitement liés à la forme de militantisme à l'œuvre dans RESF, un militantisme de solidarité fondé sur le parrainage et rendant possible une intégration par le bas des sans-papiers. Cette prévalence du soutien pose enfin la question des relations entre TIC et politiques de représentation.
\end{abstract}

Mots-clés: Sans-papiers, TIC, téléphone portable, internet, listes de discussion, politique de représentation, RESF, solidarité, parrainage, militantisme de solidarité, militantisme en réseau.

\section{Abstract}

This article deals with uses of the Internet - in particular discussion lists - in the Education Without Borders Network (Réseau Éducation sans Frontières, RESF), a network dedicated to supporting illegal migrants (" sans-papiers »). These uses are closely connected to the kind of activism at work within RESF, a solidarity activism based on sponsorship and which enables a bottom-up integration of the "sans-papiers ». Finally, this prevalence of support raises the issue of the relation between ICT and politics of representation.

Keywords: Sans-papiers, ICT, mobile phone, Internet, discussion lists, politics of representation, solidarity activism, patronage, network activism.

\section{Resumen}

El presente artículo tiene por objeto los usos de Internet, y, en especial, de las listas de discusión en la Red Educación sin Fronteras (RESF), red de apoyo a los sin papeles. Estos usos están estrechamente ligados al militantismo que caracteriza a dicha red - militantismo de solidaridad basado en el apadrinamiento y que permite una integración por lo bajo de los 
TIC et parrainage dans les mouvements militants de défense des sans-papiers en France

sin papeles. Este tipo de apoyo suscita la cuestión sobre las relaciones entre las TIC y las políticas de representación.

Palabras clave : Los sin papeles, TIC, telefonía móvil, Internet, foros de discusión, política de representación, RESF, solidaridad, apadrinamiento, militantismo de solidaridad, militantismo reticular. 


\section{Introduction : Intégration par le haut et intégration par le bas}

Lorsque l'on parle en France d'intégration des migrants, que ce soit dans le langage politique ou dans celui des sciences humaines, on la conçoit le plus souvent en termes d'insertion ou d'hospitalité institutionnelle. Elle renvoie dès lors à un ensemble de procédures économiques, juridiques et politiques de gestion des populations immigrées dans laquelle l'État joue le rôle principal. Cette intégration est fonction de "systèmes ": système social, système d'éducation, système de santé... Cependant, cette intégration n'est pas le tout de l'intégration, elle est ce que l'on peut appeler une intégration par le haut.

À côté d'elle, sur ses marges, en fonction de ses manques, de ses absences, et parfois aussi en rupture avec les politiques d'immigration, se développent des formes multiples d'intégration par le bas, une hospitalité (Gotman, 2001) qu'on pourrait dire relationnelle en ce sens qu'elle repose avant tout sur des rapports entre individus, rapports de proximité affective, d'amitié, sur des sentiments de solidarité. L'intégration ne passe plus tant alors par une série d'étapes, de paliers que par un accompagnement quotidien, une aide circonstanciée, une série de " petits " gestes de soutien par des individus qu'on peut appeler des parrains de migrants. "Pour un migrant, partager le carnet d'adresse de "mon Français", utiliser son compte pour héberger des chèques de paiement, acheter un téléphone mobile avec son aide, sont autant des preuves d'hospitalité que des pas vers l'intégration » (Diminescu, 2005).

Une telle forme d'intégration n'aura pas attendu l'émergence des technologies de l'information et de la communication (TIC) pour se manifester. II n'en reste pas moins que les TIC, en tant qu'elles participent d'une prolifération des vecteurs et formes de communication et de relation, sont susceptibles de jouer un rôle d'accélérateur, de démultiplicateur des " gestes » de l'intégration par le bas.

C'est ce que nous allons ici tâcher de démontrer à partir de l'exemple du Réseau Éducation sans frontières (RESF), réseau de soutien et de solidarité en faveur des sans-papiers. Nous débuterons en situant les activités du réseau du point de vue historique, tout d'abord en faisant un retour sur les usages du téléphone portable dans les luttes des sans-papiers de la fin des années 1990 et en revenant sur un passé plus lointain encore avec l'appropriation et le détournement de la technique de communication radiophonique lors de la lutte 
pour l'indépendance algérienne à la fin des années 1950. Puis, nous situerons les activités de RESF du point de vue politique, à travers les transformations du militantisme, plus particulièrement le passage à un "militantisme en réseau ", étroitement allié à un " militantisme de solidarité ", s'adossant sur des pratiques des TIC. Nous présenterons ensuite RESF, en portant notre attention sur la structuration, les modes d'organisation et de participation au réseau, avant de rendre compte des usages des TIC et plus particulièrement des listes de discussion sur internet comme outil de mobilisation, de diffusion d'alertes, comme medium de réflexion. Nous prolongerons nos interrogations en nous intéressant au parrainage en tant que modèle du lien entre membres de RESF et sans-papiers, en tant qu'incarnation des valeurs de solidarité et d'hospitalité prônées par le réseau. Enfin, nous serons alors en mesure de soulever la question des politiques de représentation, soit "parler pour » (un autre) soit " parler en son nom propre ", à l'œuvre dans RESF en lien avec les pratiques des TIC.

\section{Le téléphone portable comme arme dans la lutte des sans-papiers}

Le 28 juin 1996, l'église Saint-Bernard à Paris fut occupée par plus de 300 migrants irréguliers, se donnant eux-mêmes pour nom celui de sans-papiers. Cette dénomination relève d'une stratégie consistant à rejeter les représentations de l'illégalité, le statut de « hors-la-loi », en leur substituant la problématique du «titre » (des papiers), ceci afin de pointer la contradiction ${ }^{1}$ d'une exclusion de fait avec un droit égal à la présence, à l'inclusion. Le droit que les sans-papiers revendiquaient était un droit à la visibilité et à la parole, autrement dit les droits de toute démocratie. Ce que démontraient les sanspapiers, ainsi que l'affirmait Étienne Balibar dans un texte lu le 25 mars 1997 en I'honneur des "sans-papiers de Saint-Bernard », c'est que la démocratie est " une institution du débat collectif, mais dont les conditions ne sont jamais données d'en haut. Toujours il faut que les intéressés conquièrent le droit à la parole, la visibilité, la crédibilité, courant le risque de la répression. [...] La citoyenneté [...] n'est pas une institution ou un statut, mais une pratique collective ». Exclus parmi les exclus, subalternes, les « sans-papiers ont cessé de figurer simplement des victimes pour devenir des acteurs de la politique

\footnotetext{
${ }^{1}$ Cette contradiction est nécessitée et reproduite sans cesse par la logique et la politique de ce qu'Emmanuel Terray appelle la « délocalisation sur place », c'est-à-dire la reconstitution « dans nos propres villes, dans nos propres pays [des] conditions qui sont celles de la main-d'œuvre dans les pays du Tiers Monde " (Terray, 2008). (Voir également Balibar, Chemillier-Gendreau, CostaLascoux et Terray, 1999).
} 
démocratique » (Balibar, 1998, p.25). Pour affirmer leur droit à la visibilité, ils se sont exposés et ont agi ; pour affirmer leur droit à la parole, ils ont élevé leur voix, ont fait entendre la voix des sans-voix.

Dans cette prise de parole, les technologies mobiles ont joué un rôle décisif. Le téléphone portable, dont la diffusion était alors beaucoup moins importante qu'elle ne l'est aujourd'hui, est devenu une arme collective ${ }^{2}$, un vecteur de négociation avec les pouvoirs publics, un instrument de communication avec la presse, mais aussi une source potentielle de conflit interne, en ce que, parfois monopolisé par les délégués, il se transformait en symbole de pouvoir individuel ${ }^{3}$. Ceci est parfaitement illustré par Madjiguène Cissé, déléguée et porte-parole du "collectif Saint-Bernard», dans son livre Paroles de sanspapiers (on notera l'insistance sur l'acte de parole) et, plus particulièrement, dans un chapitre intitulé "Le poulet et le portable » où elle relève l'hybridation, au sein du mouvement des sans-papiers, des pratiques animistes et de l'usage des technologies de pointe, en premier lieu du téléphone portable, "signe distinctif du porte-parole des sans-papiers". Madjiguène Cissé évoque une scène traumatisante. Arrêtée après l'invasion de l'église par la police, elle fut déshabillée par des femmes policiers devant sa fille. Les sarcasmes des policières, disant "Ah! Ah! Elle ne l'a plus son portable, la porte-parole », conférèrent un sens particulier à cette humiliation; ils signifiaient qu'en tant qu'étrangère, en tant qu'Africaine, "à peine descendue de son arbre ", elle ne pouvait légitimement s'approprier un tel symbole de la modernité, ou sinon, sur le seul mode de l'imitation (Cissé, 1998, p.107). Après l'humiliation et le dénudement, l'arrachement de cette arme de lutte qu'était le portable signait le déni du droit à la parole et par conséquent de la citoyenneté, un retour pour les sans-voix à une invisibilité qui n'est pas sans rappeler celle de l'homme invisible - l'Africain-Américain - que décrit Ralph Ellison dans son roman (Ellison, 1995). Humiliation physique, discrimination raciale et culturelle, violence dans les politiques de représentation ne sont ainsi que les trois faces d'une même et unique exclusion.

Nous devons ici, pour reprendre une expression de Homi K. Bhabha (Bhabha, 1995), nous «rappeler» de Frantz Fanon. Dans un des essais

\footnotetext{
${ }^{2}$ C'est le syndicat Sud PTT qui avait distribué des téléphones portables aux délégués des sanspapiers.

«Dans le creuset des mouvements des sans-papiers, la dimension stratégique du téléphone portable a commencé à fonctionner une fois que le syndicat Sud-PTT a apporté son aide et doté les délégués d'un téléphone portable, à la place des talkie-walkie utilisés dans la coordination traditionnelle des manifestations de rue. Au-delà de l'aspect organisationnel, cet instrument est devenu le médium par lequel plusieurs négociations avec les pouvoirs publics ont eu lieu, et par lequel le contact avec la presse est demeuré ouvert. C'est également par ce biais que les mesures efficaces ont été prises dans les moments de tension » (Diminescu, 2002).
} 
TIC et parrainage dans les mouvements militants de défense des sans-papiers en France

composant L'an $V$ de la révolution algérienne, intitulé " Ici la voix de l'Algérie ", un titre à nouveau très significatif, Fanon met en relief la conjugaison de l'élévation de la voix des sans-voix avec l'appropriation ou le détournement des techniques, en l'occurrence de la technique radiophonique, pendant la guerre de libération nationale du peuple algérien colonisé. II n'y a néanmoins aucune apologie de la technique chez Fanon. Certes, il affirme qu'« en tant que technique instrumentale au sens restreint, le poste de TSF développe les pouvoirs sensoriels, intellectuels et musculaires de l'homme dans une société donnée " (Fanon, 2001, p.55). Mais, il sait également que, dans la situation coloniale, détenir un poste de radio c'est non seulement témoigner de son appartenance à la petite bourgeoisie occidentale, mais c'est aussi « sentir vivre et palpiter la société coloniale ", et, pour un Algérien, se soumettre à la voix de l'autre, de l'occupant, une voix française qui ne s'adresse qu'aux Français. En ce sens, le refus de la pénétration de cette technique au sein de la société algérienne ne saurait s'expliquer par l'attachement à la tradition; elle ne saurait être une forme de contre-acculturation. Ce refus est bien plutôt une stratégie de repli, de résistance, de contre-assimilation, nécessaire bien qu'encore purement passive, improductive.

Ce n'est qu'à partir du moment où s'engage la lutte de libération nationale, au moment où les colonisés éprouvent le besoin de posséder leurs propres sources d'informations, que ces défenses tombent, que la radio est spontanément adoptée et qu'est créée «La voix de l'Algérie ». Cette appropriation, c'est alors bien celle d'un instrument de lutte du colonisateur retourné contre lui-même, c'est une subversion. En ce sens, elle ne saurait relever de la répétition des pratiques du colonisateur. D'autant plus que la guerre des ondes, le brouillage des diffusions ennemies, produit des phénomènes originaux. La " Voix », devenue presque inaudible, exige un travail d'élaboration collectif de l'information par des auditeurs sommés de se prononcer sur ce qui a été dit ou non, des auditeurs qui deviennent non seulement des interprètes mais plus encore des vecteurs et des auteurs du discours, des porte-paroles. C'est pourquoi Fanon préfère au terme d'appropriation de la technique celui de "quasi-invention », indissociable de la création d'une communauté en acte.

Entre ce schéma de la "quasi-invention » dévoilé par Fanon et les usages du téléphone portable par les sans-papiers de l'église Saint-Bernard, les analogies sont certaines. Ce que l'on observe dans les deux cas, c'est d'abord l'usage de la technique en tant qu'arme collective, un outil de mobilisation, d'organisation et d'unification, fondé sur son détournement au nom de revendications de groupes en quête d'une "simple » existence politique. Les pratiques de l'internet sont susceptibles de prolonger ce détournement. Peut- 
être même l'intensifient-t-elles singulièrement dans la mesure où, ainsi que nous allons le voir, elles se présentent " en quelque sorte comme la traduction technologique de l'engagement caractéristique du "néo-militantisme" " (Granjon, 2002/2003, p.12), militantisme en réseau dont nous allons brièvement indiquer les spécificités.

\title{
2. Militantisme en réseau, militantisme de solidarité et TIC
}

L'émergence d'internet a contribué à la genèse et au développement de nouvelles formes de critique sociale. Les TIC se sont avérées parfaitement adaptées à cette critique que Fabien Granjon définit de la manière suivante :

\begin{abstract}
"Ce sont de nouvelles formes d'engagement militant qui apparaissent ainsi, décrivant le passage d'un militantisme "traditionnel" à un engagement distancié (un néo-militantisme, une "nouvelle" critique sociale) dont les symptômes se lisent tout autant dans le renouvellement des modes d'action collective que dans les formes de sociabilité qu'il convoque par ailleurs. Le trait principal de cette évolution est sans aucun doute la perte d'influence de la forme fédérale au profit d'un modèle d'organisation en réseau. Les néo-militants s'expriment alors de préférence au travers de multiples voies, plus modestes et chacune consacrée à une cause particulière. L'engagement se négocie davantage dans la multiplicité de projets revendicatifs menés de front à un niveau collectif ou individuel. Ce qui compte avant tout pour le néomilitant, c'est de s'inscrire non plus au sein d'une organisation idéologique clairement identifiée mais plutôt de générer des projets ou de s'intégrer à des projets initiés par d'autres et d'exploiter toutes les connexions susceptibles de s'avérer à cet égard utiles » (Granjon, 2002 ; voir aussi Granjon, 2001).
\end{abstract}

Internet est un outil majeur pour les acteurs de ce nouveau militantisme. Apparaissent de nouvelles figures de la critique sociale qui se présentent comme autant de facilitateurs de réseaux. Ce sont tout d'abord les "passeurs " 
TIC et parrainage dans les mouvements militants de défense des sans-papiers en France

dont la première fonction est « de faire circuler sur le réseau des réseaux des informations plus ou moins informelles qui s'élaborent au sein de cercles militants restreints »; pour les passeurs «l'engagement en ligne ne se dissocie pas de l'expérience vécue ». Viennent ensuite les «filtreurs » qui cherchent à " soulager les militants-internautes menacés par l'inflation des données et leur faciliter la gestion et l'appropriation de l'information transmise ", les principes et normes de sélection étant définis collectivement et répondant à une logique d'auto-organisation. II y a enfin les « interprètes ", dont la participation peut être exclusivement "en ligne» et qui se présentent comme des experts "pourvoyeurs de sens » fournissant autant d'outils de réflexion à l'usage des militants (Granjon, 2002).

La combinaison de l'engagement offline et online autorise ce que Jacques Ion avait appelé le « militantisme post-it » opposé au « militantisme de la carte » et " offrant aux militants engagés dans une organisation très présente en ligne la possibilité de se retirer momentanément de l'action tout en restant informés de ce qui se passe, et donc de pouvoir se réengager sans difficultés quand ils le désirent de nouveau » (Granjon, 2005, p.25). L'utilisation des outils de communication facilite en outre la diffusion des savoirs, la création d'un capital de connaissances, l'émergence d'une mémoire des luttes.

Ces remarques s'avèrent plus décisives encore lorsque l'on sait que le « militantisme en réseau » ne se situe plus tant dans le champ de la production et de l'économie que "dans le champ de la culture, de la sociabilité ou des valeurs "; ses acteurs ont "pour objectif de répondre à des situations d'exclusion qui ont pour point commun une relégation sociale qui n'est plus forcément en rapport avec des conflits de classe » (Granjon, 2002/3, p.14). Le néo-militantisme a notamment pris en charge " un type de critique sociale liée aux question de précarité - c'est toute la thématique des "sans", sans-papiers, sans-droits... » (Granjon, 2005, p.24).

Le «militantisme en réseau» s'est ainsi allié à un "militantisme de solidarité ». Ainsi que le rappelle Johanna Siméant, "les mobilisations "de" sans-papiers, précisément, ne sont jamais uniquement des mobilisations de sans-papiers. Elles sont tout autant le fait de populations que nous qualifierons de "soutiens" " (Siméant, 1998, p.25). Siméant définit le "militantisme de solidarité » comme "une forme spécifique de militantisme défendant des personnes, des malheureux qui ne sont pas a priori les mêmes que ceux qui soutiennent leur cause »(Siméant, 1998, p.64), et mentionne en particulier l'antiracisme et les mouvements en faveur des immigrés.

Les mouvements de et en faveur des sans-papiers constituent donc un terrain privilégié pour l'observation de l'usage des TIC dans les pratiques 
militantes. Ils rendent également possible une meilleure compréhension des relations entre cet usage et les questions d'intégration des migrants. Nous allons montrer qu'ils nous obligent à repenser l'intégration comme intégration par le bas ou, pour reprendre à nouveau les termes de Siméant, "insertion dans des réseaux sociaux spécifiques " (Siméant, 1998, p.41), ou plus précisément selon nous par des réseaux sociaux spécifiques.

\section{Qu'est-ce que le Réseau Éducation sans frontières?}

Le Réseau Éducation sans frontières (RESF) a été créé fin juin 2004 suite à une réunion tenue à la Bourse du Travail et réunissant enseignants et personnels de l'Éducation nationale, parents d'élèves, éducateurs, associations, syndicats et organismes de défense des droits de l'Homme ${ }^{4}$. Tous étaient alarmés par la situation d'enfants et adolescents sans papiers scolarisés et ils décidèrent de créer ce qu'ils appelèrent un " réseau de solidarité » appelant à la vigilance, à la circulation d'informations et à la mobilisation en faveur de la régularisation de ces enfants.

En France, la scolarisation d'un enfant interdit toute « reconduite à la frontière », autrement dit toute expulsion. Mais, si jamais durant cette scolarité, l'enfant sans-papiers n'a pas été régularisé, qu'adviendra-t-il de lui dès lors qu'il sera en âge de devenir un " candidat » à l'expulsion ? Même si cette dernière se fait attendre, demeure l'angoisse permanente d'une interpellation pouvant forclore toute perspective future. Leurs parents sans-papiers, eux, sont en permanence sous la menace d'une "Obligation de quitter le territoire français » (OQTF). Or, le « départ» des parents est bien entendu le plus souvent synonyme du départ des enfants. La loi française, censée protéger les familles de la rupture, de la séparation, prend ici une signification pour le moins originale: la cellule familiale est préservée... par le départ de tous ses membres.

On comprend dès lors que l'action de RESF ne saurait se limiter aux enfants, qu'elle concerne très souvent des familles entières et qu'en ce sens, c'est une lutte générale pour les sans-papiers qui est ainsi engagée. Ces actions sont diverses: sensibilisation, diffusion d'informations, manifestations et mobilisations en faveur d'individus et de familles, parrainages d'enfants sanspapiers et aide juridique, pression auprès des autorités publiques et intervention lors des interpellations de la police, les « rafles », afin de prévenir la population

\footnotetext{
${ }^{4}$ http://www.educationsansfrontieres.org/?article5
} 
TIC et parrainage dans les mouvements militants de défense des sans-papiers en France

et déceler d'éventuels vices de formes. II est nécessaire de souligner pour terminer que le combat de RESF est un combat pour des individus en un sens déjà intégrés, puisque ce sont des personnes scolarisées.

RESF est organisé en collectifs, ce terme devant être entendu en son sens le plus large, à savoir comme un " ensemble de personnes travaillant ensemble, une organisation de fait et non de droit ${ }^{5}$ \%. Certes, RESF inclut des organisations au sens fort, associations, syndicats... mais «la participation individuelle est très importante dans le réseau qui est avant tout composé d'individus". "Le réseau RESF fonctionne essentiellement sur les individualités, sur les relations ${ }^{6} »$. Les membres de RESF ne connaissent en général que très peu d'autres membres ; ils en ont rencontré en face à face un plus petit nombre encore. Personne ne sait réellement combien de personnes participent à chacun des collectifs $^{7}$, les niveaux d'implication dans le réseau étant également très variables. Les collectifs RESF s'étendent sur tout le territoire français, mais aussi en Belgique et au Maroc ${ }^{8}$. Chacun d'eux diffuse ses propres informations, a ses propres contacts, ses propres listes de discussion et dans certains cas, son propre site web.

La nature même des collectifs détermine leurs relations. La structuration du réseau est profondément originale. Ainsi que le précise l'un de ses membres actifs, " ce n'est pas une organisation hiérarchique, "en verticalité", mais une structure informelle, sans statuts, sans organes, sans permanents, sans bureaux ${ }^{9} »$. Michel Élie, membre lui aussi de RESF (resf34, Héraut) et l'un des fondateurs de l'Observatoire des Usages de l'internet (OUI), affirme que la seule force propre au réseau réside dans "l'émotion suscitée qui saisit le milieu scolaire, et bien au-delà, quand un jeune ou sa famille dévoile sa situation et les risques encourus " (Élie, 2006). Cependant, la force de l'émotion et de l'indignation ne se traduit pas en pure spontanéité, en "horizontalité », en absence d'organisation. Le réseau possède un bureau national, des antennes par région, par ville et éventuellement par arrondissement, des comités de soutien par groupe d'écoles, d'autres types de collectifs pouvant encore s'agréger au réseau. Chaque collectif a ses propres principes d'organisation, leur coordination supposant des interactions permanentes. Nous sommes en

\footnotetext{
${ }^{5}$ Entretien avec Sylvie, administratrice de la liste de discussion sur internet resf75 (Paris), 10 juillet 2008.

${ }_{7}^{6} \mathrm{Ibid}$.

7 Seul les administrateurs des listes de discussion sur internet ont en quelque sorte une vue générale des collectifs.

${ }^{8}$ Voir http://www.educationsansfrontieres.org/

${ }^{9}$ Entretien avec Jean-Jacques, membre de RESF, 27 novembre 2007.
} 
présence d'un fonctionnement dit "en transversalité », d'un réseau ouvert. RESF est « un être associatif et politique d'un genre nouveau ${ }^{10}$ ".

Évoquer les premiers contacts des acteurs de RESF avec le réseau et la naissance de leur engagement, c'est faire face à une multiplicité d'occasions, de circonstances, de motifs individuels ${ }^{11}$. Ceci nous rappelle comme nous le disions que RESF est organisé en collectifs composés avant tout d'individus. II ne faut cependant pas mésestimer le rôle des associations ou syndicats dans un tel contact. Mais, si c'est l'organisation elle-même qui s'engage parfois dans RESF, notamment par la création d'un collectif, l'engagement naît plus régulièrement des liens d'amitié au sein de l'organisation ou encore par une "démarche individuelle ». L'organisation est avant tout en ce cas le lieu d'une activité militante reposant sur un engagement personnel. Ce sont par ailleurs souvent des liens de proximité, d'amitié, et plus généralement le « ouï dire » qui sont les vecteurs de l'engagement. Le rôle de la proximité, notamment affective, est plus évident encore, quand il s'agit de proximité avec les sans-papiers eux-mêmes. C'est pourquoi les membres de RESF sont très souvent des enseignants ou des parents d'élèves, témoins des menaces d'expulsions d'enfants sans-papiers scolarisés.

\section{RESF et les TIC : les listes de discussion sur internet}

Dans la mesure où RESF est composé de collectifs structurés de manière non-hiérarchique, son organisation couvrant un vaste territoire, la coordination des activités "locales» et nationales (voire internationales) ne pouvaient manquer de trouver dans les TIC « un outil idéal dont l'utilisation paraît facile et peu onéreuse » (Granjon, 2005, p.29 ; voir aussi Diminescu, 2002). Plus encore, on peut formuler l'hypothèse que cette organisation exigeait, pour mener à bien son action, un usage étendu des TIC.

Internet s'est présenté pour RESF comme un amplificateur, comme un levier permettant d'atteindre rapidement une très large couverture géographique, d'obtenir le soutien de syndicats enseignants, d'associations spécialisées dans le droit des étrangers, et avant tout de multiples individus pour lesquels la participation sur internet a pu parfois constituer la première forme d'engagement, celle-ci se prolongeant sur le terrain. II a permis de diffuser

\footnotetext{
${ }^{10}$ Entretien avec Jean-Jacques, membre de RESF, 27 novembre 2007.

${ }^{11}$ Les données suivantes sont extraites des réponses (25 répondants) à un questionnaire diffusé sur la liste de discussion resf75 en juillet 2008.
} 
TIC et parrainage dans les mouvements militants de défense des sans-papiers en France

l'action des collectifs locaux à travers notamment le partage d'expériences sur des cas pratiques. II a rendu possible une meilleure circulation de l'information et une meilleure connaissance par chacun des textes officiels.

On touche là à l'un des aspects essentiels du militantisme animé par les TIC, à savoir la constitution à travers les interactions en ligne de bases de connaissances pratiques, d'une mémoire interne "somme de tous les savoirs de toutes sortes accumulés pour leur donner forme et mutualisés en ligne [...]. Avec le Net, cette mémoire se trouve distribuée et partagée de manière beaucoup plus large " (Granjon, 2005, p.26). Internet, en tant qu'outil de diffusion des savoirs, facilite par ailleurs le développement de véritables expertises qui étaient nécessaires par exemple lorsque RESF a déplacé la lutte sur le terrain administratif et juridique ou encore a mis en place des systèmes de parrainage républicain. RESF a créé sur internet une véritable «base de connaissances de cas pratiques ${ }^{12} »$.

L'outil « liste de discussion » s'est révélé être pour RESF un instrument de lutte essentiel, parfaitement adapté au fonctionnement en « réseau ouvert ${ }^{13}$ ».

De quoi parle-t-on sur ces listes? On peut distinguer huit catégories de messages $^{14}$ :

- Discussions et réflexions sur les textes de lois, politiques d'immigration: elles constituent une part importante des échanges sur la liste, bien que difficilement quantifiable.

- Discussions et conseils sur des cas pratiques: c'est dans ces discussions que se mutualisent les connaissances, que se développe une expertise, notamment juridique, en matière d'immigration.

- Alertes, soutien et mobilisation en faveur d'un sans-papiers donné, actions " anti-rafle »: les alertes constituent une part extrêmement importante des échanges sur la liste ; elles permettent une diffusion « instantanée » de l'information rendant possible une mobilisation rapide. Internet est néanmoins souvent en ce cas relayé par le téléphone portable ${ }^{15}$.

\footnotetext{
${ }^{12}$ Entretien avec Sylvie, administratrice de la liste de discussion sur internet resf75 (Paris), 10 juillet 2008.

${ }^{13}$ Entretien avec Jean-Jacques, membre de RESF, 27 novembre 2007.

${ }^{14}$ Ce découpage est fondé sur l'observation des échanges sur la liste de discussion resf75 de septembre 2007 à septembre 2008.

${ }^{15}$ Entretien avec Jean-Jacques, membre de RESF, 27 novembre 2007.
} 
- Pétitions : la circulation et la signature de pétitions constituent l'une des activités les plus régulières au sein du réseau.

- Organisation et informations sur les manifestations, rassemblements : comme dans toute activité militante, les manifestations et rassemblements de soutien sont un élément essentiel de RESF. La liste permet de prévoir, organiser et, surtout, de diffuser l'information à ce sujet.

- Organisation du réseau, réunions, planning: la liste de discussion permet également d'assurer l'organisation du réseau, de prévoir des réunions, de diffuser des plannings.

- Polémique : la liste de discussion est parfois le lieu de polémiques touchant par exemple au processus de décision au sein de RESF.

- Sujet en marge : ce sont des discussions portant sur des sujets aux frontières des problématiques de RESF : par exemple les questions de logement des migrants.

Les listes de discussion favorisent la coordination des collectifs, le passage de l'interaction à l'action collective. Les réponses des membres de RESF à notre questionnaire, et en particulier à la question des différentes activités menées, nous ont permis d'observer que, pour la majorité de ces membres, les pratiques online et offline sont profondément mêlées. Ainsi, $85 \%$ des répondants disent participer régulièrement aux actions de terrain (manifestations, mobilisations...) tandis que $75 \%$ d'entre eux participent régulièrement (online) à la discussion de cas pratiques et $60 \%$ à la réflexion sur les textes de lois, politiques d'immigration ${ }^{16} \ldots$ II ne semble donc pas y avoir de partage, au sein du réseau, entre des individus qui mèneraient une activité exclusivement ou presque online et d'autres qui privilégieraient le terrain ${ }^{17}$.

Si nous désirons à présent comprendre comment les pratiques des TIC au sein de RESF s'articulent avec la promotion de formes d'intégration par le bas, il nous faut porter une attention toute particulière au parrainage dans la mesure où celui-ci se présente comme la plus fondamentale formalisation des rapports de solidarité.

\footnotetext{
${ }^{16}$ La circulation des pétitions demeure l'activité la plus régulière pour $90 \%$ des répondants ; ceci n'est pas étonnant dans la mesure où c'est une activité demandant un investissement assez faible. 17 Nous n'évoquons pas ici le rôle joué par le téléphone portable, notamment les téléphones d'urgence, confiés tour à tour à des membres de RESF assurant une présence et un rôle de diffusion de l'information en cas d'alertes : arrestation d'un sans-papiers, annonces d'une "rafle » de sans-papiers.
} 
TIC et parrainage dans les mouvements militants de défense des sans-papiers en France

\title{
5. Le parrainage
}

Le parrainage dans RESF désigne en premier lieu ce qu'on appelle parrainage républicain des sans-papiers. Celui-ci a exactement la même origine et la même valeur que le parrainage civil (le baptême civil relève du même principe), variante non-religieuse de la cérémonie au cours de laquelle une, deux ou plusieurs personnes sont choisies afin d'apporter à l'enfant un éventuel soutien moral et pratique. Le parrainage républicain n'a pas de valeur légale :

\begin{abstract}
«Au fondement juridique incertain, situé entre le décret du 26 juin 1792, la loi du 20 septembre 1792 et le décret du 8 juin 1794, le parrainage républicain n'est prévu par aucun texte législatif. Le maire ou un autre officier d'état civil habilité n'est pas tenu de le célébrer, le parrainage n'étant pas acte d'état civil, de même qu'il n'y a aucun cérémonial préétabli. Les parrains et marraines prennent uniquement un engagement de valeur morale, les documents ou certificats délivrés pour l'occasion n'ayant aucune valeur juridique. II ne faut en effet pas confondre la cérémonie de parrainage républicain et la désignation d'un tuteur légal. Le parrainage républicain ou le baptême civil vise seulement à faire rentrer symboliquement le parrainé dans la communauté républicaine et le faire adhérer à ses valeurs » (Julliard, 2008, pp.5-6).
\end{abstract}

Néanmoins, les parrainés ou filleuls sans-papiers disposent d'un livret de parrainage qui, en tant que signe même de soutien par des individus de la société d'accueil, protège les sans-papiers. Le parrainage est en quelque sorte un bouclier; il fournit une protection qui n'est pas de l'ordre du droit mais de l'ordre des rapports de forces entre des personnes dotées de prérogatives. C'est pourquoi il est important d'impliquer des représentants de l'État. L'aspect symbolique du parrainage est essentiel : il est important qu'il ait lieu dans une mairie, ou éventuellement dans une école dans la mesure où celle-ci « représente » la citoyenneté.

Le parrain est le plus souvent un individu qui connaît personnellement un enfant sans-papiers : ce peut être un enseignant, un parent d'élève. Ce peut 
être également quelqu'un qui désire être parrain sans connaître de sans-papiers et qui est mis en relation avec un sans-papiers. Qu'il soit antérieur ou postérieur à la cérémonie, le rapport affectif, voire l'amitié, est un facteur essentiel du parrainage. "Ce qui compte, c'est la création de liens plus que la cérémonie elle-même ${ }^{18}$ ». Le parrain est celui qui est prêt à " donner un coup de main pour tout » à son filleul, au-delà des démarches de régularisation. "Le parrainage est d'abord une question de petites "choses"19 " : c'est un soutien quotidien au parrainé. Ainsi le parrainage républicain relève bien de ce qu'on a appelé l'intégration par le bas. Celle-ci ne s'oppose pas ici à l'intégration par le haut. Au contraire, on a vu qu'elle employait les symboles mêmes de l'intégration institutionnelle, étatique.

Cependant, en dehors du parrainage républicain, officiel, ou encore formel, de nombreux membres de RESF s'occupent personnellement de dossiers de sans-papiers. C'est ainsi tout un pan des relations entre membres de RESF et sans-papiers qui relève du parrainage au sens large, entendu comme solidarité, relation affective de personne à personne se traduisant par toute une série de gestes d'hospitalité. Ce modèle de relation, fondement de RESF, est menacé car identifié par les autorités à un délit : le fameux « délit de solidarité ». De plus en plus, la presse détaille des cas de sanctions auxquelles des citoyens et militants s'exposent lorsqu'ils s'avisent d'apporter un soutien ou simplement de protester contre les procédures d'expulsion engagées contre un étranger en situation irrégulière.

L'activité principale des parrains consiste sans aucun doute dans l'aide aux démarches pour la régularisation, l'obtention des papiers: aide auprès des administrations, constitution de dossiers, accompagnement en préfecture pour la demande de titres de séjour. C'est là la mission première des parrains qui se confond en un certain sens avec leur rôle de «bouclier». Une autre activité fondamentale est le soutien en cas d'urgence : par exemple le recours devant le tribunal administratif en cas de refus de titres de séjours assortis de mesures d'éloignement (Obligation de quitter le territoire français, Arrêté préfectoral de reconduite à la frontière). Cette activité consiste en une série d'interventions auprès des préfectures, commissariats, voire auprès du gouvernement français. Viennent enfin d'autres formes de soutien : aide à l'hébergement, suivi scolaire, veille, aide à la recherche d'emploi occasionnel, apprentissage du français, notamment après la régularisation, soutien psychologique et moral.

\footnotetext{
${ }^{18}$ Entretien avec Sylvie, administratrice de la liste de discussion sur internet resf75 (Paris), 10 juillet 2008.

${ }_{19}$ Ibid.
} 
TIC et parrainage dans les mouvements militants de défense des sans-papiers en France

Le modèle du parrainage gouverne de façon très nette les modalités de participation et de nombreux échanges au sein des listes de discussion. On peut ainsi observer ${ }^{20}$ que tant qu'un individu n'est pas immédiatement menacé, il est pris en charge par un membre de RESF, son "parrain », qui peut s'adresser aux abonnés de la liste de discussion afin d'obtenir des conseils pratiques, notamment au sujet de la régularisation. Lorsque la situation devient plus grave, plus urgente pour cet individu, un nombre croissant de membres de la liste en viennent à participer aux interactions avec diffusion de l'information sur d'autres listes RESF, voire d'autres listes hors RESF, le parrain dirigeant souvent les échanges et organisant la mobilisation ${ }^{21}$.

Reste à présent à se poser la question de l'usage des TIC par les sanspapiers eux-mêmes. Cette question nous intéresse dans la mesure où elle permet de reformuler le problème des politiques de représentation des subalternes.

\section{Les sans-papiers et les TIC}

Quels sont donc les usages que les sans-papiers eux-mêmes font des TIC au sein de RESF ? Pour répondre à cette question, il est nécessaire de rendre compte dans un premier temps de leur participation aux actions en dehors (offline) du réseau. II y a une présence occasionnelle voire régulière des sanspapiers aux rassemblements, manifestations ou autres évènements locaux organisés par RESF. Une telle participation demeure cependant " périlleuse » dans la mesure où les sans-papiers se retrouvent ainsi exposés à de potentielles arrestations; elle est donc nécessairement limitée par le risque encouru. La participation des sans-papiers aux réunions du réseau est quant à elle rare, voire nulle, ceci pour la majorité des collectifs. Enfin, la solidarité des sans-papiers à l'égard de la lutte est parfois conçue par les membres de RESF comme un mode de «participation » au réseau. En ce sens, on pourrait affirmer

\footnotetext{
${ }^{20}$ Les analyses qui suivent sont fondées sur le traitement des messages de la liste de discussion resf75 sur une période d'un an. Nous avons extrait automatiquement (à l'aide du logiciel Mimetic développé par Christophe d'Iribarne que nous remercions à cette occasion) les noms de sanspapiers des titres des messages ceci afin d'analyser les relations entre membres de RESF (émetteurs de messages) et les sans-papiers au sujet desquels ils s'expriment, au nom desquels ils agissent. Nous avons par ailleurs extrait l'ensemble des messages relatifs à quelques cas de sanspapiers afin d'étudier l'évolution de ces cas, la chronologie de la participation des différents membres.

${ }^{21}$ Certains cas de sans-papiers peuvent enfin devenir des " cas historiques » en raison de leur durée, de leur complexité, de la tragédie qui les accompagne. Ce n'est qu'alors, dans une certaine mesure, que la figure du parrain tend en quelque sorte à s'effacer.
} 
que le soutien se renverse, ou plutôt que la solidarité est réciproque : les sanspapiers appuient ceux-là mêmes qui les soutiennent.

II faut à présent dire que la participation des sans-papiers aux activités online, en particulier à la liste de discussion est également très faible, voire nulle, à quelques exceptions près. Pour ce qui est des modes de communication entre parrains et leurs protégés, la plupart des échanges se font en face à face ou par téléphone portable; la messagerie électronique est relativement peu employée. L'une des raisons à cela est le privilège conféré par les sans-papiers eux-mêmes à la rencontre en face à face ${ }^{22}$. En effet, pour des individus menacés, la présence sur internet peut être ressentie comme une forme d'exposition. II peut y avoir une crainte de s'exprimer. La rencontre physique permet au contraire "de savoir qui on a en face de $\operatorname{soi}^{23}$ ". II est cependant évident que ce privilège repose aussi sur de potentielles insuffisances dans la connaissance de la langue française. Plus généralement, c'est le capital financier et culturel des sans-papiers qui est en cause : "Pour contribuer par exemple à une liste de diffusion, il faut d'abord un certain capital financier, pour s'équiper en ordinateur et en logiciels, et s'offrir un abonnement à un fournisseur d'accès ; il faut disposer aussi d'un capital culturel conséquent, savoir se servir d'une machine, savoir écrire, savoir lire, savoir chercher de l'information ; enfin, il faut posséder des compétences techniques assez précises, et savoir utiliser les nouvelles technologies » (Granjon, 2005, p.29).

\section{TIC et politiques de représentation}

On a vu précédemment que les TIC jouaient, d'une part, un rôle essentiel dans les activités militantes de RESF, en tant qu'outil d'organisation et de mobilisation en urgence, et, d'autre part, qu'elles étaient le plus souvent absentes, et au mieux secondaires, dans les pratiques des sans-papiers euxmêmes ${ }^{24}$. Les questions d'accès ou de capital financier et culturel n'expliquent pas tout. Si le réseau veut être un soutien pour les sans-papiers, fait-il de ces derniers, ainsi que se le demande un de nos répondants, de véritables partenaires?

\footnotetext{
${ }^{22}$ Entretien avec Sylvie, administratrice de la liste de discussion sur internet resf75 (Paris), 10 juillet 2008.

${ }^{23}$ Ibid.

24 À la différence de nombreux réseaux d'exilés politiques (Palestiniens, Tunisiens, Chinois) s'appuyant sur les compétences professionnelles de leurs membres, en particulier de leurs informaticiens, qui sont donc peu affectés par la fracture numérique.
} 
TIC et parrainage dans les mouvements militants de défense des sans-papiers en France

N'existe-t-il pas en effet des différences essentielles entre l'occupation de l'église Saint-Bernard par les sans-papiers et la mobilisation au sein du réseau RESF en faveur des sans-papiers ? Pour le dire simplement, dans le premier cas, les sans-voix élevaient la voix, tandis que dans le second, des membres de la société civile agissent au nom des sans voix. La question des politiques de représentation est ainsi posée. Quant à la "quasi-invention » de la technique pensée par Fanon, elle ne semble plus être l'œuvre des subalternes eux-mêmes mais de ceux qui les représentent. Pour résumer, ne serait-ce pas parce que les membres de RESF entendent parler et agir pour les sans-papiers et non aider à ce que les sans-papiers parlent en leur nom propre, que les listes de discussion sur internet peuvent être un instrument majeur de la lutte sans être investies par les sans-papiers eux-mêmes?

En ce sens, il serait peut être nécessaire de s'interroger sur ce que Aris Papatheorodou appelle le fantasme d'une nouvelle démocratie promise par internet. "La chose reste à démontrer, car cela resterait une démocratie d'initiés. Cela pose problème par exemple pour un mouvement comme celui des sans-papiers : la majorité des gens qui s'expriment sur leur liste de discussion sont plutôt finalement leurs soutiens » (Papatheorodou, 2005, p.61). On pourrait ainsi dire qu'encore aveugle aux formes d'exclusion numérique, le réseau RESF se présentant comme ardent défenseur et promoteur de la démocratie, continuerait à œuvrer dans un espace où les subalternes n'ont pas de voix, pas de parole.

Cependant, vouloir à tout prix que le subalterne se représente, ne risque-t-il pas d'impliquer, dans certaines situations, d'œuvrer pour la préservation de la subalternité elle-même ? C'est ce qu'affirme Gayatri C. Spivak: "Qui diable veut protéger la subalternité ? [...] Aucun militant ne veut garder le subalterne dans l'espace de la différence [...]. Vous ne donnez pas la parole au subalterne. Vous travaillez pour le foutu subalterne, vous travaillez contre la subalternité » (Spivak, 1992, p.46). En ce sens, l'opposition des deux formes de représentation n'est peut-être qu'un voile masquant des problèmes bien plus essentiels.

Johanna Siméant souligne en un sens analogue qu'il y a un danger à vouloir ériger systématiquement les sans-papiers en tant que purs sujets, ou inversement en purs objets, des revendications. Pour parer à ce danger, il est nécessaire de comprendre que le militantisme de solidarité ne peut se penser en posant un " dedans ", « impliquant les acteurs sociaux ayant le plus intérêt à y participer » : les sans-papiers, et un "dehors » de la mobilisation impliquant " des "soutiens désintéressés" ou "moraux" » (Siméant, 1998, p.25).

Dans le cas de RESF, maintenir l'opposition du « parler pour » et du « parler en son nom propre ", c'est oblitérer la forme même des relations entre membres 


\section{Dana DIMINESCU et Matthieu RENAULT}

de RESF et sans-papiers, à savoir le parrainage, sans lequel on ne saurait comprendre les politiques de représentation mises en œuvre. Le parrainage, au sens large des relations affectives et de solidarité de personne à personne et qui se présente comme un paradigme de l'intégration par le bas, oblige à dépasser l'opposition des deux formes de représentation.

Ce que promeut le parrainage c'est un " parler et agir avec ». La première fonction du parrain est non de se substituer au sans-papiers dans ses démarches de régularisation, d'agir pour lui, mais bien plutôt de l'accompagner, de l'aider, d'agir avec lui. Tel est le sens profond de la notion de solidarité. II n'y a en fait guère que lors des arrestations, des recours aux tribunaux, que les membres de RESF parlent et agissent pour les sans-papiers. Mais, on comprend bien que ce moment, c'est justement celui où le sans-papiers se retrouve privé de toute possibilité d'action, de parole.

C'est donc en fonction de ce modèle de l'« avec », du parrainage, qu'il faut interpréter l'usage des TIC au sein de RESF ; opposer usage de la part des membres de RESF et non-usage de la part des sans-papiers est insuffisant car c'est ignorer les relations qui unissent les premiers aux seconds. II faudrait bien plutôt dire que les membres de RESF jouent le rôle d'écrivain public pour les sans-papiers : ils ne formulent pas tant le contenu d'un message qu'ils aident à le structurer, à l'organiser, à le mettre en forme, à le faire entendre. Lorsque Hannah Arendt affirmait que le sans-droits ${ }^{25}$ était celui ou celle qui était privé d'un droit à la parole, elle ne voulait certes pas dire qu'ils n'avaient pas la capacité de parler mais plutôt qu'on leur refusait la possibilité que leurs paroles aient un sens, qu'elles soient entendues (Arendt, 2006) ${ }^{26}$. Les sans-papiers ne sont pas en ce sens étrangers à l'usage des TIC au sein de RESF : ils sont en un certain sens les co-auteurs des messages bien qu'ils n'en soient pas les porteurs.

\footnotetext{
${ }^{25}$ Les sans-papiers se qualifient eux-mêmes régulièrement de sans-droits.

${ }^{26}$ Les positions de Gayatri C. Spivak, dans son article, "Can the Subaltern Speak? " (Spivak, 1988), sont de ce point de vue assez proches de celles de Arendt.
} 
TIC et parrainage dans les mouvements militants de défense des sans-papiers en France

\section{Références bibliographiques}

ARENDT H., 2006, Les origines du totalitarisme, tome 2 : L'impérialisme, Paris, Seuil.

BALIBAR É., 1998, "Ce que nous devons aux "Sans-Papiers" » dans Droit de cité, Éditions de l'Aube.

BALIBAR É., M. CHEMILLIER GENDREAU, J. COSTA-LASCOUX et E. TERRAY, 1999, Sans-papiers : l'archaïsme fatal, Paris, La Découverte.

BHABHA H. K., 1994, "Remembering Fanon: self, psyche and the colonial condition ", dans P. WILLIAMS et L. CHISMAN (dir), Colonial discourse and post-colonial theory: a reader, New York, Columbia University Press, pp.112-125.

CISSÉ M., 1998, Parole de sans-papiers, La Dispute, Paris.

DIMINESCU D., 2002, "L'usage du téléphone portable par les migrants en situation précaire ", Hommes et migrations, $n^{\circ} 1240$, " migrants.com »; http://www.ticm.msh-paris.fr/spip.php?article26, dernière consultation le 10 juin 2009.

DIMINESCU D., 2005, "Le migrant connecté », Migrations/Société, vol.17, n¹02, pp. 275-292, http://www.ticm.msh-paris.fr/spip.php?article32, dernière consultation le 10 juin 2009.

ÉLIE M., 2006, Le RESF : un réseau " citoyen » influent fondé sur des usages de l'internet: http://www.oui.net/modules/news/article.php?storyid=121, dernière consultation le 10 juin 2009.

ELLISON R., 1995, Invisible Man, New York, Vintage International.

FANON F., 2001, "Ici la voix de l'Algérie », dans L'an V de la révolution algérienne, Paris, La Découverte.

GOTMAN A., 2001, Le sens de l'hospitalité, Essai sur les fondements sociaux de l'accueil de l'autre, Paris, Presses universitaires de France.

GRANJON F., 2001, L'internet militant : Mouvement social et usage des réseaux télématiques, Rennes, Apogée.

GRANJON F., 2002, «Les militants-internautes: passeurs, filtreurs et interprètes ", Communication (Université Laval. Département d'information et 
de communication), vol.22, $\mathrm{n}^{\circ} 1$, pp.11-32, http://multitudes.samizdat.net/Lesmilitants-internautes-passeurs, dernière consultation le 10 juin 2009.

GRANJON F., 2002/3, «Les répertoires d'action télématiques du néomilitantisme ", Le mouvement social, $\mathrm{n}^{\circ} 200$.

GRANJON F., 2005, " L'internet militant, entretien avec Fabien Granjon », Matériaux pour l'histoire de notre temps, juillet-septembre, $\mathrm{n}^{\circ} 79$.

JULLIARD E., 2008, Les parrainages républicains de sans-papiers, 2005-2008: entre soutien aux sans-papiers et construction du pouvoir politique local Étude de cas dans le $13^{e}$ arrondissement de Paris, Mémoire de Master 1 Science politique, Dir. J. Valluy, Université Panthéon-Sorbonne (Paris I), http://terra.rezo.net/IMG/doc/JULLIARD Emilien.doc, dernière consultation le 10 juin 2009.

PAPATHEORODOU A., 2005, "Samizdat.net, l'histoire d'un projet de médias alternatifs sur l'internet", Matériaux pour l'histoire de notre temps, juilletseptembre, $\mathrm{n}^{\circ} 79$.

SIMÉANT J., 1998, La cause des sans-papiers, Paris, Presses de Sciences Po.

SPIVAK G. C., 1988, "Can the subaltern speak? ", dans C. NELSON et L. GROSSBERG (dir.), Marxism and interpretation of culture, Champaign, University of Illinois Press, pp.271-315.

SPIVAK G. C., 1992, "Interview with Gayatri Chakravorty Spivak : New nation writers conference in South Africa », Ariel : A Review of International English Literature, 23(3), pp.29-47.

TERRAY E., 2008, "Délocalisation sur place, libre circulation et droits des migrants, Entretien avec Emmanuel Terray, suivi d'une réflexion sur les "politiques mémorielles" ", Culture \& Révolution, 15 avril, http://culture.revolution.free.fr/en question/2008-04-15-E-Terray.html, dernière consultation le 10 juin 2009. 